\title{
THE STRUCTURE OF THE ENVELOPES OF SYMBIOTIC NOVAE ${ }^{+}$
}

\section{G.B. BARATTA ${ }^{\prime}$, A. DAMINELI NETO ${ }^{2}$, C. ROSSI $^{3}$, R. VIOTTI $^{2}$}

(1) Osservatorio Astronomico, Via del Parco Mellini 84, 00136 Roma

(2) Istituto Astrofisica Spaziale, CNR, Via E. Fermi 21, 00044 Frascati RM

(3) Istituto Astronomico, Università La Sapienza, Via Lancisi 29, 00161 Roma

(*) On leave of absence from Instituto Astronomico e Geofisico da Universidade de Sao Paulo, Sao Paulo, Brasil.

(+) Based on spectra collected at the Dominion Astrophysical observatory, Victoria, Canada, and at the Laboratorio Nacional de Astrofisica, Minas Gerais, Brasil.

Symbiotic novae are composite spectrum stars whose light history is characterized by one single major outburst. At high resolution their optical and UV spectra are very rich of emission lines belonging to a wide ionization range. Generally one can identify both narrow and broad line systems. The latter includes emission lines with multiple structure, broad emission wings, WR features, and P Cygni lines. Frequently the broad line profiles are largely variable with time. They should be associated with the major stellar outburst. An overview of the problem with extensive references can be found in Viotti (1989).

The earlier 1969 observations of V1329 Cyg have shown that the strongest hydrogen lines, HeII 4686, and the forbidden OIII and NeIII display a multiple structure with many components with radial velocities from -320 to $+300 \mathrm{~km} / \mathrm{s}$ (Crampton et al. 1970; Baratta and Viotti 1989, Fig.1). These features are probably formed in low density cloudlets ejected in different directions during the 1966 outburst. This multiple structure lasted for many years after the outburst with large profile variations (Wallerstein et al. 1989) which can be attributed to the 950d orbital motion of the system and to partial obscuration of some components by dense and inhomogeneous circumstellar gas or dust. The profile is quite reminiscent of that observed in classical novae, except for the lower velocity range. Fig.2 shows the profile of [OIII] in Nova Cen 1986. The lines are characterized by a multiple structure with components' radial velocity ranging from -1300 to $+1100 \mathrm{~km} / \mathrm{s}(\mathrm{Fig} .3)$. In this case the components' distribution may suggest ejection in preferred directions, rather than with spherical symmetry.

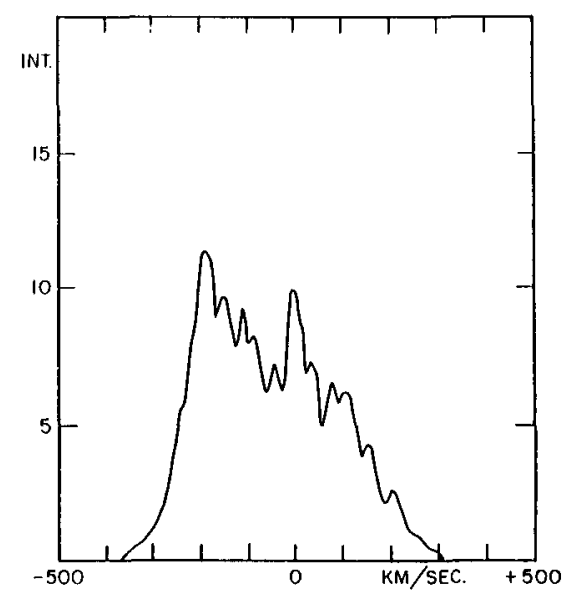

Fig.1. The mean profile of the [NeIII] and [OIII]lines in V1329 Cyg in 1969. The radial velocity of the emission components ranges from -320 to $+300 \mathrm{~km} / \mathrm{s}$ (Dominion Astrophysical Observatory, Crampton et al. 1970). 
WR features have been observed in V1329 Cyg, HM Sge, and RR Tel during the earlier phases of their spectral evolution. They might be originated in a transient hot high velocity wind from the exploded degenerated component of the system. The wind should also be responsible of the broad wings observed in the strongest UV emission lines in RR Tel and AG Peg.

$\underline{P}$ Cygni profiles have been observed during different activity phases of symbiotic stars. In 1950 the HeI 3888 line in AG Peg developed multiple absorption components with velocities from -72 to $-382 \mathrm{~km} / \mathrm{s}$, which later disappeared. Even larger velocities $(-685$ and $-865 \mathrm{~km} / \mathrm{s})$ were observed in the $1951-52$ spectra of $R R$ Tel. Broad $P$ Cygni profiles with variable shape are displaced by CIV and NV line in the UV spectrum of AG Peg. This again conflem the presence of variable hot winds in symbiotic novae, also long after their outburst.

Finally, as concerns the narrow lines high resolution measurements have shown that their FWHM generally increases with I.P. from few to several $10 \mathrm{~km} / \mathrm{s}$, suggesting a stratification of the narrow line formation region. This is possible related to line formed at different depths in the cool giant wind.

\section{REFERENCES}

Baratta, G.B., Viotti, R.: 1989, Astron. Astrophys. in press.

Crampton, D., Grygar, J., Kohoutek, L., Viotti, R.: 1970, Astrophys. Lett. $\underline{6}, 5$. Viotti, R.: 1989, in Cataclysmic and Related Variables, M. Hack ed., NASA in press. Wallerstein, G., Solf, J., Mikolajewska, J., Crampton, D., Brugel, E.: 1989, P.A.S.P. 101, 189 .

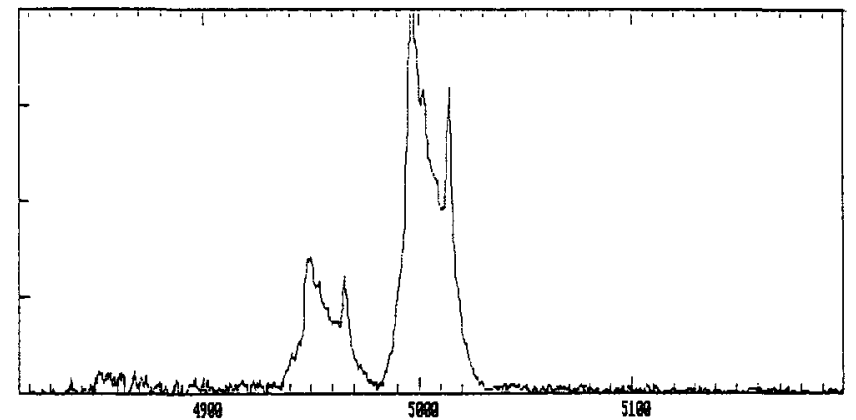

Fig.2. The region near $5000 \mathrm{~A}$ in Nova Cen 1986 on 10 Apri1 1988 (collected with the $160 \mathrm{~cm}$ telescope of the Laboratorio Nacional de Astrofisica, Minas Gerais, Brasil).

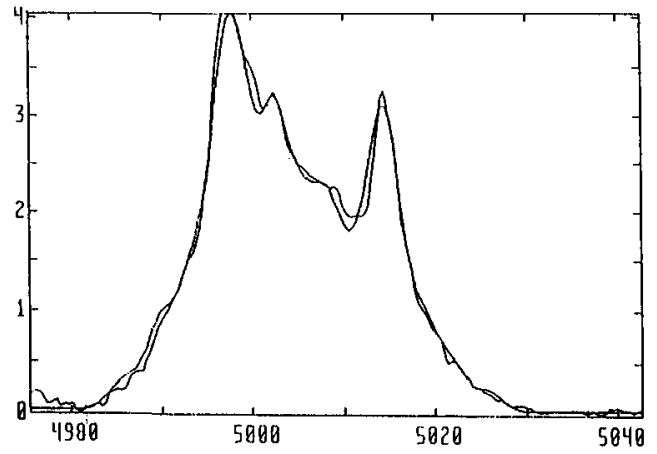

Fig.3. The [OIII] 5007 A line in Nova Cen 1986 fitted with 16 gaussians, from -1300 to $+1100 \mathrm{~km} / \mathrm{s}$, and FWHM of 3.3 to $4.2 \mathrm{~A}$ (main peaks are at -620 and $+460 \mathrm{~km} / \mathrm{s}$ ). 